\title{
Dual Tensor Atlas Generation Based on a Cohort of Coregistered non-HARDI Datasets
}

\author{
Matthan Caan ${ }^{1,2}$, Caroline Sage ${ }^{3}$, Maaike van der Graaf ${ }^{1}$, \\ Cornelis Grimbergen ${ }^{1}$, Stefan Sunaert ${ }^{3}$, Lucas van Vliet ${ }^{2}$, and Frans Vos ${ }^{1,2}$ \\ 1 Dept of Radiology, Academic Medical Center, University of Amsterdam, NL \\ 2 Quantitative Imaging Group, Delft University of Technology, NL \\ 3 Dept of Radiology, University Hospitals of the Catholic University of Leuven, BE \\ m.w.a.caan@tudelft.nl
}

\begin{abstract}
We propose a method to create a dual tensor atlas from multiple coregistered non-HARDI datasets. Increased angular resolution is ensured by random variations of subject positioning in the scanner and different local rotations applied during coregistration resulting in dispersed gradient directions. Simulations incorporating residual coregistration misalignments show that using 10 subjects should already double the angular resolution, even at a relatively low $b$-value of $b=1000 \mathrm{smm}^{-2}$. Commisural corpus callosum fibers reconstructed by our method closely approximated those found in a HARDI dataset.
\end{abstract}

\section{Introduction}

The white matter (WM) structure of the human brain can be studied by means of Diffusion Weighted Magnetic Resonance Imaging (DW-MRI). The diffusion is measured in multiple directions, from which the principal diffusion orientation field is reconstructed [1. Fiber tracking throughout this field allows reconstruction of WM bundles, such as the corpus callosum [2].

The validity of local comparison of WM properties between different cohorts depends on good inter-subject anatomical correspondence. Variability in anatomical correspondence can be minimized by coregistration of the data on voxel basis. Both an affine and a non-rigid transformation can be computed to compensate for global and local variability, respectively [3].

Coregistration of data requires an appropriate reference space to be chosen. The reference space to which the subjects are transformed, can either be represented by one subject out of the cohort, or by an atlas that is built by averaging coregistered datasets. The ICBM FA atlas was generated by averaging 81 affinely transformed DTI datasets [4]. Recently, several DTI atlas-building approaches have been proposed with some yielding single subject-based atlases, e.g. [5], and others yielding population-based atlases [6].

By coregistering the data to such an atlas, fiber tracking can be performed in atlas space, which allows comparing tract statistics between patient and control groups [7]. However, tracking is known to be biased in regions where fibers are crossing. Fiber tracts may either deviate or truncate in such regions, due to 
inadequate modeling of the diffusion process by a single tensor. Fiber tracking through complex tissue regions is facilitated by higher order diffusion models, such as a dual tensor model or q-ball [89. In order to apply such complex models, High Angular Resolution Diffusion Imaging (HARDI) data needs to be acquired [10]. One of the properties of a HARDI-acquisition is a high number of gradient directions, being more than 100. Clinical data is generally sampled at 6-45 gradient directions.

The design of a comparative study to find structural changes along fibers may be regarded as sampling a high-dimensional space, spanned by respectively three spatial coordinates, gradient directions and included subjects. Let us assume that the amount of available scanning time is constant, such as in a large population study in which only a few minutes of acquisition time is reserved for DW-MRI. In order to reduce bias in fiber tracking, one might prefer to acquire HARDIdata, yielding a denser sampling along the gradient axes. A fixed-time constraint implicitly imposes a lower sampling density along either the spatial or subject axes. Both are generally unacceptable, since not only small anatomical structures but also statistical power in the comparison need to be retained. Therefore, acquiring HARDI-data for clinical studies is not always realistic, as scanning time needs to be increased or scanner hardware needs to be upgraded to facilitate a higher SNR. Also, ongoing studies with conventional DW acquisition protocols, may not comply with the previously mentioned criteria.

In this study, we propose a method to fit a higher order diffusion model to a cohort of coregistered non-HARDI data. We consider the diffusion attenuation profiles of multiple subjects as realizations of one underlying fiber distribution. After non-rigid coregistration of the datasets, the resulting signal profiles are gathered to generate an artificial HARDI-dataset (disregarding the low $b$-value), with a high sampling density along the gradient axes. First, simulations incorporating residual coregistration misalignments are used to demonstrate the potential increase in angular resolution. Second, a dual tensor model is fitted to the data to generate a dual tensor atlas. We expect that in this atlas, tracking will be as accurate as in HARDI-data.

\section{Method}

\subsection{Dual Tensor Model}

We propose to estimate a dual tensor model from non-HARDI data of multiple subjects, with the purpose of precisely estimating two independent diffusion orientations per voxel. We assert a model for the diffusion weighted signal $S_{j}\left(\mathbf{q}_{\mathbf{j}}\right)$ of a subject $j$, with two tensors combined with an isotropic compartment:

$$
\frac{S_{j}\left(\mathbf{q}_{\mathbf{j}}\right)}{S_{j, 0}}=f_{1} \exp \left(-\mathbf{q}_{\mathbf{j}}^{\mathbf{T}} \mathbf{D}_{\mathbf{1}} \mathbf{q}_{\mathbf{j}}\right)+\left(1-f_{1}-f_{i s o}\right) \exp \left(\mathbf{q}_{\mathbf{j}}^{\mathbf{T}} \mathbf{D}_{\mathbf{2}} \mathbf{q}_{\mathbf{j}}\right)+f_{i s o} \exp \left(-\mathbf{q}_{\mathbf{j}}^{\mathbf{T}} \mathbf{q}_{\mathbf{j}} D_{i s o}\right) .
$$

Here $S_{j, 0}$ is the signal for subject $j$ measured without diffusion weighting and $f_{\text {... }}$ are the normalized volume fractions, while $D_{i s o}$ is the isotropic diffusion 
constant. The vector $\mathbf{q}_{\mathbf{j}}=\sqrt{b} \mathbf{g}_{\mathbf{j}}$ reflects the diffusion weighting $b$ in gradient direction $\mathbf{g}_{\mathbf{j}}$. The diffusion tensors are spectrally decomposed, $\mathbf{D}_{\mathbf{1 , 2}}=\mathbf{R}_{\mathbf{1 , 2}} \mathbf{E R}_{\mathbf{1 , 2}}$, with $\mathbf{E}=\operatorname{diag}\left(\lambda_{\|} \lambda_{\perp} \lambda_{\perp}\right)$ being the eigenvalue matrix with axial and planar diffusion values (assuming axially symmetric tensors). The two rotation matrices $\mathbf{R}_{\mathbf{1 , 2}}$ are parametrized using Euler angles $\alpha_{1-4}$. The vector of eight parameter values $\boldsymbol{\theta}=\left(\lambda_{\|} \lambda_{\perp} \alpha_{1-4} f_{1} f_{\text {iso }}\right)$ is estimated in a least-squares sense.

\subsection{Simulating Variation in the Cohort}

A cohort of subjects that is studied for epidemiological processes or pathology is carefully defined by setting precise inclusion criteria. Thus, undesirable variations unrelated to the process of interest are minimized and may include handedness, sex, age and education [11. Remaining variations are to be seen as normally distributed axial and planar diffusion values. Anatomical variations between subjects are apparent as differences in size and position of WM bundles. Non-rigid coregistration of the data accounts for the majority of these differences, residual misalignment of the principal diffusion direction was found to be $20^{\circ}$ [12]13.

In an experiment on synthetic data, the expected angular resolution (i.e. the absolute error in the angle between the major axes of the tensors) will be computed, as a function of the number of included datasets. The abovementioned variations are incorporated by generating a signal profile per subject with normally perturbed eigenvalues and angles (using equation 1). Also, Rician noise is included to distort the simulated data. Gradient directions are randomly rotated per subject (see section 2.3). The parameter vector $\boldsymbol{\theta}$ is subsequently estimated on the combined data of multiple subjects. In this experiment, a diffusion weighting of $b=1000 \mathrm{smm}^{-2}$ is used to reflect our conventional clinical protocol.

\subsection{Building a Dual Tensor Atlas}

Coregistration. The DWI datasets of multiple subjects need to be transformed to a common reference space. In this study, a population-based atlas is build. To generate this atlas, all datasets are first affinely coregistered to the ICBM FA template [4]. As an intermediate step, we construct a (single tensor) DTI atlas that contains the full diffusion information [6]. The original DWIs were coregistered to our DTI atlas using an algorithm based on a viscous fluid model and optimization of mutual information as the coregistration criterion [12. This coregistration consists of a combination of an affine and a non-rigid transformation to correct for global and local morphological differences, respectively. The effect of deformation discontinuities is removed by smoothing of the final deformation field with a Gaussian kernel of FWHM $=3 \mathrm{~mm}$.

Transformation. A diffusion-weighted image describes the MR signal attenuation due to diffusion in a specific gradient direction. If it is rotated, in our case during registration, signal correspondence with the gradient direction needs to be retained. This was done previously by fitting a single tensor to the data, after which preservation of principal direction (PPD) was applied to realign tensors 
with the underlying fiber structure after transforming the tensor field [12]. The DWIs were then recomputed from the rotated tensor field. In the latter step, the number of degrees of freedom (DOF) is reduced to six (i.e. the DOF of the single tensor model), which prohibits a higher order model fit to the data. We therefore transform the original DWIs without intensity correction. Instead, the gradient directions are rotated, such that the vectors $\mathbf{q}_{\mathbf{j}}$ become:

$$
\mathbf{q}_{\mathbf{j}}=\sqrt{b} \cdot \mathbf{R}_{\text {nonrigid }, \mathbf{j}}(x, y, z) \cdot \mathbf{R}_{\text {affine, } \mathbf{j}} \cdot \mathbf{g}_{\mathbf{j}} \text {. }
$$

The rotational component of the affine transformation $R_{\text {affine, } \mathbf{j}}$ is applied globally, while the rotation due to non-rigid transformation $\mathbf{R}_{\text {nonrigid,j }}(x, y, z)$ is applied per voxel $(x, y, z)$.

An advantage of our reorientation method is that both $R_{\text {affine,j }}$ and $\mathbf{R}_{\text {nonrigid,j }}$ will be dispersed among subjects (as demonstrated in figure 2(c)). Patient positioning in the scanner and inter-subject variations in bundle trajectories contribute to this distribution. The combined set of multiple subjects is sampled at a higher angular resolution than the original acquisition protocol. Hence, the merged dataset not only has an increased SNR, but indeed approaches a HARDI-dataset due to random dispersion of gradient directions per subject after coregistration.

Tensor Estimation and Fiber Tracking. The dual tensor model can now be estimated (equation 11) on the merged dataset, using the dispersed sampling of gathered gradient directions. The least squares difference of the model and data is minimized using Levenberg-Marquardt optimization. Error-function parametrization ensures positiveness of the parameters. Next, WM fibers are reconstructed using High Angular Fiber Tracking (HAFT) [14. As an extension to common streamline tracking algorithms, this method generates branches of fibers if within a voxel the angle between both tensors exceeds a certain threshold. One seed Region of Interest (ROI) and one AND-ROI through which fibers should pass have to be defined.

\section{Results}

\subsection{Synthetic Data}

Synthetic data was generated according to the model in equation 1 . The eigenvalues were $\lambda_{\|}=1.5 \cdot 10^{-3} \mathrm{~mm}^{2} \mathrm{~s}^{-1}$ and $\lambda_{\perp}=0.4 \cdot 10^{-3} \mathrm{~mm}^{2} \mathrm{~s}^{-1}$ [15] and the angle between both tensors was $45^{\circ}$. For second and further realizations, the eigenvalues and the angle were normally perturbed with a standard deviation of $10 \%$. $S_{0}$ was set to 230 , whereas a volume fraction $f_{1}=0.5$ was used. Sixteen icosahedric and dodecahedric gradient directions on a half sphere [16] and along the 16 corresponding, antipodal directions were chosen, conform to our clinical scanning protocol. Rician noise $(\mathrm{SNR}=20: 1)$ distorted the data.

Next, data of different numbers of datasets with dispersed gradient directions were gathered and the dual tensor model was fit to the data. The isotropic volume fraction $f_{\text {iso }}$ was included to account for inter-subject diffusion variations, 


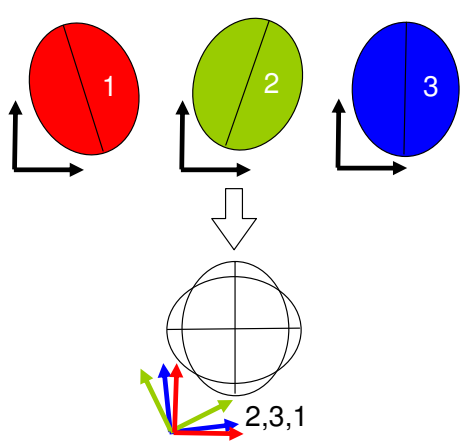

(a)

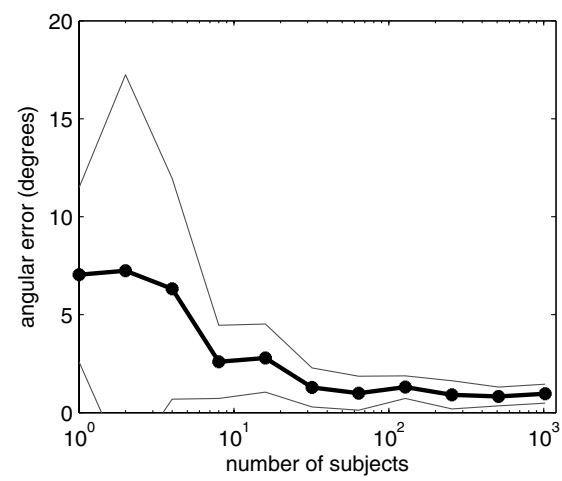

(b)

Fig. 1. (a) Illustration of the dual tensor atlas generation, the gradient directions are randomly dispersed after coregistration, such that a dual tensor fit becomes possible. (b) Mean angular error (bold) with standard deviation (gray) in degrees as a function of the number of included datasets. The two tensors per subject were slightly perturbed, such that the angles were normally distributed with $\sigma=5^{\circ}$. The average angle between both tensors was $45^{\circ}, 32$ random gradient directions were used per subject.

whereas the isotropic diffusion was set constant: $D_{\text {iso }}=0.5 \cdot 10^{-3} \mathrm{~mm}^{2} \mathrm{~s}^{-1}$. The mean and standard deviation of the angular error (defined in section 2.2) as a function of the number of subjects are depicted in figure 1. Initially, both the mean error and the standard deviation remained high when data of up to four subjects were used. For small sample sizes, modeled residual misalignments induce a slight fitting instability. Adding more subjects lowered the mean error and the standard deviation to $2^{\circ} \pm 1^{\circ}$ for 64 subjects. Further increase of data did not result in a lower error, again due to the applied perturbations.

This experiment shows that the angular resolution may be increased by estimating tensors based on a cohort.

\subsection{Clinical Data}

The proposed method was applied to a small cohort of 11 healthy subjects, of which DTI data were acquired on a 3.0T scanner (Intera, Philips, Best, The Netherlands). The spatial resolution was $2.0 \times 2.0 \times 2.2 \mathrm{~mm}$, such that 64 axial slices of matrix size $128 \times 128$ were acquired, while 32 gradient directions were used (see section 3.1) with a diffusion weighting of $b=1000 \mathrm{smm}^{-2}$. Additionally, one set of images was acquired without any diffusion-weighting. Eddy current distortions were corrected for by an affine registration in the phase direction [17. For comparison, a HARDI-dataset was acquired, with 92 gradient directions and $b=1800 \mathrm{smm}^{-2}$. The acquired datasets were coregistered as described in section 2.3. The DWIs were resampled without intensity correction, and diffusion weighting vectors $\mathbf{q}_{\mathbf{j}}$ were rotated (equation 2) and stored per voxel per deformation field of a subject. The merged dataset in total contained $32 \cdot 11=352$ DWIs. 


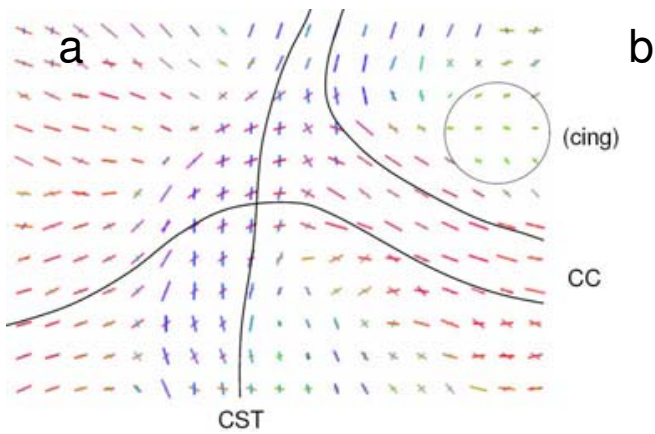

b
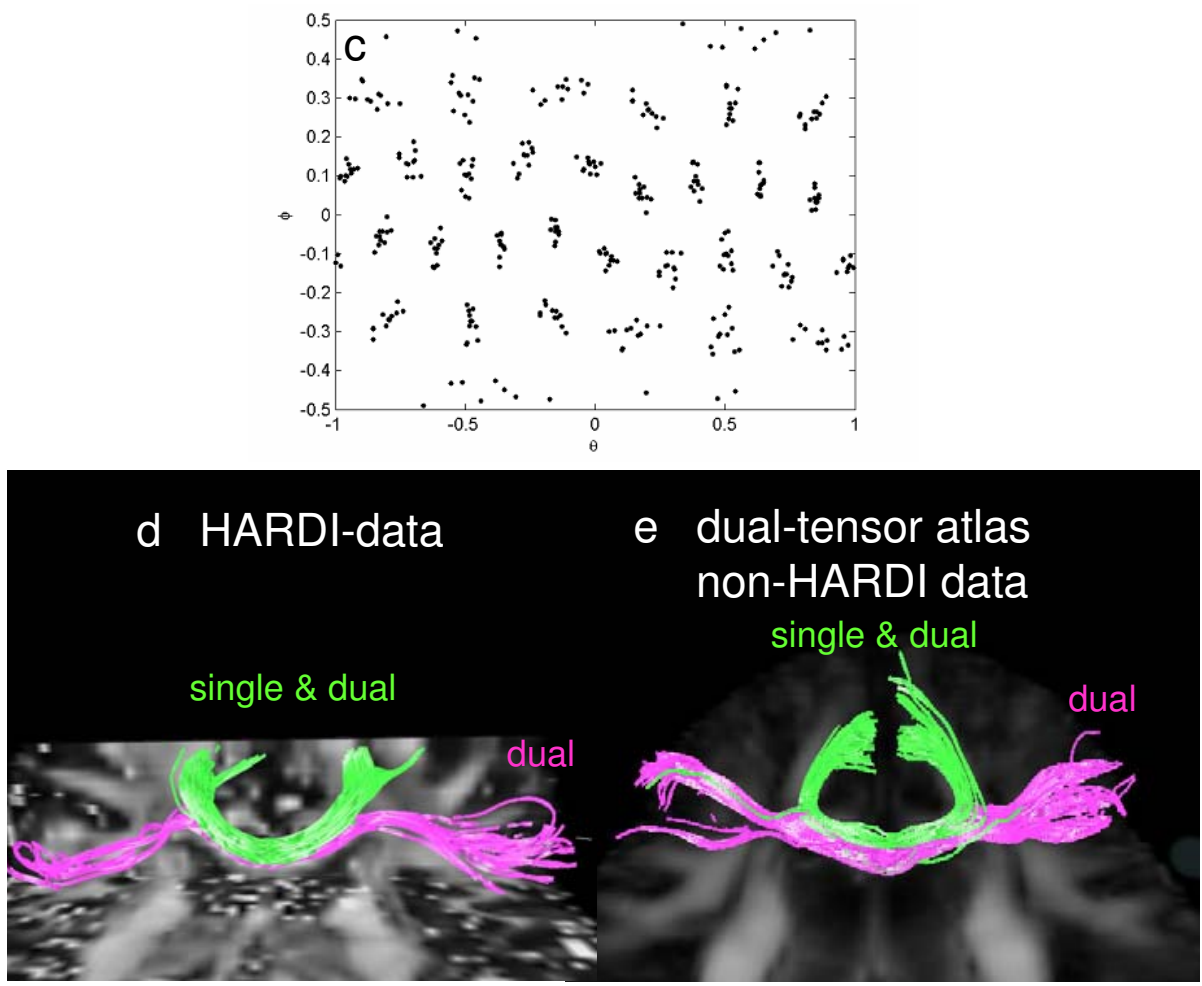

Fig. 2. Dual tensor atlas results. (a) Coronal cross-section through the estimated dual tensor atlas field, with principal diffusion orientations per voxel scaled by the volume fraction. Anatomical references are given $(\mathrm{CST}=$ corticospinal tract, $\mathrm{CC}=$ corpus callosum, cing $=$ cingulum) together with an impression of bundle trajectories (drawn by the authors). (b) Aggregated ADC-profile plotted as points and fitted model in gray with principal orientations. Conventional color coding is used. (c) Aggregated gradient directions plotted in spherical coordinates. (d-e) HARDI-data and dual tensor atlas tracking result: part of the corpus callosum is tracked (green fibers), as well as commissural fibers (magenta fibers). 
The dual tensor fit was initialized by positioning the principal eigenvectors in the plane of the first two eigenvalues of a single tensor fit. Dual tensor parameters were then estimated in each intercranial voxel. The results of the dual tensor atlas estimation are shown in figure 2. In figure 2(a), two distinct orientations within one voxel are mainly seen in the region where the corpus callosum is known to cross the corticospinal tract. Aggregated signal values within one voxel with the fitted model are depicted in figure 2(b). Due to the low $b$-value, low contrast is seen in the modeled signal. Moreover, significant noise and/or intersubject variation may be observed (e.g. in the extension of the blue vertical vector in figure 2(b)). This confirmed the need for a sufficient amount of data in order to precisely estimate the orientation. In fact, high inter-voxel consistency in orientation in figure 2(a) is observed, indicating high fitting stability. Unfortunately, the gradient directions were not distributed uniformly, as displayed in figure 2(c). Still, a gain in angular resolution was achieved using our approach.

Fibers were reconstructed through the crossing and are displayed in figure 2(a). The fiber branching threshold was set to $12.5^{\circ}$, the angular threshold was $25^{\circ}$ and the single tensor FA threshold 0.1. The obtained fibers in the HARDIdataset and the dual tensor atlas are shown in figures 2 (d) and (e) respectively. Both corpus callosum fibers (in green) as well as commissural fibers (in magenta) were successfully reconstructed in both datasets. A higher number of 38 commissural fibers was measured in the HARDI-dataset, compared to 11 in the dual tensor atlas. This is explained by the fact that in the HARDI-dataset a high number of fibers was running over the same trajectory.

\section{Discussion}

We proposed a method to create a dual tensor atlas from multiple coregistered non-HARDI datasets. Increased angular resolution was ensured by random variations of subject positioning in the scanner and different local rotations applied during coregistration resulting in dispersed gradient directions. Simulations showed that using 10 subjects should already double the angular resolution, even at a relatively low $b$-value of $b=1000 \mathrm{smm}^{-2}$. Experiments on clinical data revealed distinct orientations within a fiber crossing as well as inter-voxel consistency. Commisural corpus callosum fibers reconstructed by our method closely approximated those found in a HARDI dataset.

Although the adopted coregistration method uses DWIs generated with a single tensor model, as argued in section 2.3, we have apparently obtained a good correspondence in regions with crossing fibers. In a single tensor model, fiber crossings manifest themselves by an oblate spheroid. Although the oblate spheroid does not contain orientation information in the plane of the crossing, it permits accurate location of the crossing. The required orientational information for correct steering of the coregistration comes from its surrounding regions in which a single tensor model suffices. 
We aim to apply our method to a large cohort in order to create an atlas that can be made available to the neuro-imaging community. WM bundles traveling through fiber crossings can then be included in comparative studies.

\section{References}

1. Basser, P., Mattiello, J., Bihan, D.L.: Estimation of the effective self-diffusion tensor from the NMR spin echo. J. Magn. Reson. B (103), 247-254 (1994)

2. Wakana, S., Jiang, H., et al.: Fiber tract-based atlas of human white matter anatomy. Radiology 230, 77-87 (2004)

3. Maintz, J.B.A., Viergever, M.A.: A survey of medical image registration. Medical Image Analysis 2, 1-36 (1998)

4. Mori, S., Oishi, K., Jiang, H., Jiang, L., Li, X., Akhter, K., Hua, K., Faria, A.V., Mahmood, A., Woods, R., Toga, A.W., Pike, G. B., Neto, P.R., Evans, A., Zhang, J., Huang, H., Miller, M.I., van Zijl, P., Mazziotta, J.: Stereotaxic white matter atlas based on diffusion tensor imaging in an ICBM template. Neuroimage 40, 570-582 (2008)

5. Ardekani, S., Sinha, U.: Statistical representation of mean diffusivity and fractional anisotropy brain maps of normal subjects. JMRI 24, 1243-1251 (2006)

6. van Hecke, W., Sijbers, J., D'Agostino, E., Maes, F., Backer, S.D., Vandervliet, E., Parizel, P., Leemans, A.: On the construction of an inter-subject diffusion tensor magnetic resonance atlas of the healthy human brain. NeuroImage 43, 69-80 (2008)

7. Goodlett, C., Fletcher, P., Gilmore, J., Gerig, G.: Group analysis of DTI fiber tract statistics with application to neurodevelopment. NeuroImage 45, S133-S142 (2009)

8. Descoteaux, M., Deriche, R., Knosche, T.R., Anwander, A.: Deterministic and probabilistic tractography based on complex fibre orientation distributions. IEEE Trans. Med. Im. 28, 269-286 (2009)

9. Kreher, B.W., Schneider, J.F., others: Multitensor approach for analysis and tracking of complex fiber configurations. Magn. Res. Med. 54, 1216-1225 (2005)

10. Tuch, D., Weisskoff, R., Belliveau, J.W., Wedeen, V.J.: High angular resolution diffusion imaging of the human brain. In: Proc 8th ISMRM, Philadelphia, vol. 321 (1999)

11. Caan, M., Vermeer, K., van Vliet, L., Majoie, C., Peters, B., den Heeten, G., Vos, F.: Shaving diffusion tensor images in discriminant analysis: A study into schizophrenia. Medical Image Analysis 10, 841-849 (2006)

12. Van Hecke, W., Leemans, A., d'Agostino, E., De Backer, S., Vandervliet, E., Parizel, P., Sijbers, J.: Nonrigid coregistration of diffusion tensor images using a viscous fluid model and mutual information. IEEE Trans. Med. Im. 26(11), 1598 $1612(2007)$

13. Cao, Y., Miller, M., Mori, S., Winslow, R.L., Younes, L.: Diffeomorphic matching of diffusion tensor images. In: Proc. CVPR, pp. 67-76 (2006)

14. Toussaint, N., van Muiswinkel, A., Hoogenraad, F.G., Holthuizen, R., Sunaert, S.: Resolving fiber crossings: a two fiber model simulation result. In: Proc. ISMRM, vol. 13, p. 1339 (2005)

15. Pierpaoli, C., Jezzard, P., Basser, P.J., Barnett, A.: Diffusion tensor MR imaging of the human brain. Radiology 201, 637-648 (1996)

16. Akkerman, E.: Efficient measurement and calculation of MR diffusion anisotropy images using the platonic variance method. Magn. Reson. in Med. 49, 599-604 (2003)

17. Mangin, J.F., Poupon, C., Clark, C., Bihan, D.L., Bloch, I.: Eddy-current distortion correction and robust tensor estimation for MR diffusion imaging, pp. 186-193 (2006) 